\title{
FETAL ELECTROCARDIOGRAM EXTRACTION BASED ON NON-STATIONARY ICA AND WAVELET DENOISING
}

\author{
V. Vigneron ${ }^{1}$, A. Paraschiv-Ionescu ${ }^{2}$, A. Azancot ${ }^{3}$, C. Jutten $^{1}$, O. Sibony ${ }^{3}$, \\ ${ }^{1}$ LIS, INPG, 46 avenue Félix Viallet, 38031 Grenoble cedex, France, vvigne, jutten@lis . inpg. fr \\ ${ }^{2}$ STI/IGBM/LMAM, EL-Ecublens, CH-1015 Lausanne, Switzerland, ani soara . ionescu@epfl . ch \\ ${ }^{3}$ Hôpital Robert Debré, Gyn.Obs.-Exp.Fonc., 48 Bd. Serrurier, 75019 Paris, France, sibony, azancot @rdb . ap-hop-paris . fr
}

\begin{abstract}
Fetal electrocardiogram (fECG) monitoring is a technique for obtaining important information about the condition of the fetus during pregnancy and labour by measuring electrical signals generated by the fetal heart as measured from multi-channel potential recordings on the mother body surface. It is shown in this paper that the fetal ECG can be reconstructed by means of higher order statistical tools exploiting ECG non-stationarity associated with post-denoising with wavelets. The method is illustrated on real fetal ECG data.
\end{abstract}

\section{INTRODUCTION}

\section{1. fECG recording}

One interesting and difficult problem in biomedical engineering is the fetal electrocardiogram (fECG) extraction $[18,7]$. The technique was evaluated for the diagnosis of twins, fetal position [11], fetal life [16], congenital heart disease, and asphyxia. Despite this renewed promise, the difficulty of recording abdominal fECG and its questionable yield limite the clinical utility and it was used primarily for research purposes. With this technique, one can obtain fECG signals during pregnancy in a noninvasive manner, but with a weak signal-to-noise (SNR) ratio. In fact, electrodes on the maternal surface pick up the maternal ECG and, at a lower voltage, the fECG. Electrodes are also sensitive to other signals, especially electromyographic ones. Therefore, the signal observed on each electrode is a complex signal associated to a superimposition of many sources.

Although volume conduction theory presumes that the whole body is a heterogeneous conductor, the potentials created by the fetal heart are thought to be transmitted to the maternal surface mainly through the umbilical vessel-placenta pathway. Because of the above noted pecularity of fetal signal transmissions, the recording of the fECG through maternal leads to a waveform that is not affected by changing electrode location. Although the fECG wave configuration does not change when the position of the recording electrodes is changed on the surface of the mother's abdomen, the amplitude of the electrical signal may change. The idealised ECG is illustrated in Figure 1.

The shape of the fetal ECG reflects the complex electrical signal - routinely called PQRST waves - conducting within the myocardium (muscular wall of the heart) [21]. The main limitations

V. Vigneron is also with the Matisse-SAMOS UMR 8595, 90 rue de Tolbiac, 75634 Paris cedex, France. C. Jutten is professor at Ecole Polytechnique of Grenoble University. This work was partially supported by the European Union through the BLISS project, EU IST-1999-14190.

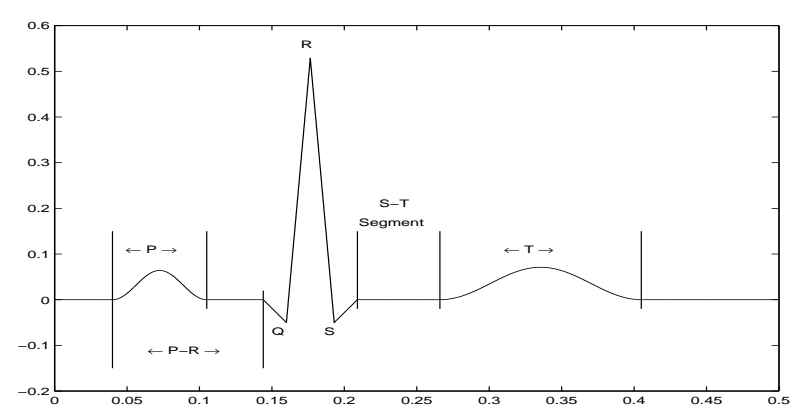

Figure 1: Decomposition of the ECG waveform.

of noninvasive fECG recording are the low amplitude signal coming from the fetal heart, the high background noise created by the maternal ECG complex, the skin potentials, electromyograms (i.e. of womb and diaphragm) and the $50 \mathrm{~Hz}$ interference. Three main characteristics should be obtained from fECG extraction for being useful in early diagnosis of cardiopathologies: fetal heart rate (FHR) [17], amplitudes of different waves, the duration of waves, segments and intervals. Scalp fECG allows for detection of the entire ECG complex, including $P, Q R S$ and $T$ waves, but are invasive. On the contrary, with external (noninvasive) fECG recording, classical signal processing methods generally detect the $R$ waves, but, due to the low SNR ratio, the fetal $P$ and $T$ waves remains hidden.

\subsection{State of the art of noninvasive fECG}

The problem was already tackled more than 50 years ago by means of now conventional adaptative noise cancelling techniques. Marvell et al. [12] studied the fECG during normal labour using computer signal averaging using cross-correlation and Fast FourierTransform (FFT). Abboud [1] shows that averaged fECG does not detect fetal arrhythmias or real time events and does not offer the tool to record in real time abdominal fECG. Blind source separation (BSS) methods [8] provide a more general framework and makes it possible to revisit such applications while expecting higher performances. Typical results are reported in De Lathauwer et al. [10], in Bacharakis et al. [2], Zarzoso [22] and in Cardoso [4]: despite signals with higher frequency than the mother heartbeats are identified to correspond to the fetal ECG, PQRST waves cannot be easily extracted. Various other reported investigations involve such cardiac signals $[9,4]$, combined with other types of 
physiological measurements, especially brain neural activity [20]. In this paper, we propose to apply recent blind separation methods for fECG extraction, (i) exploiting nonstationarity of fECG, and (ii) implementing post-denoising with wavelets, in order to try to recover the complete PQRST complex of fECG. The paper is organizes as follows. Section 2 present a theoretical sketch of BSS. Section 3 explains the basic idea of wavelet denoising. Section 4 reports the experimental results on real fECG recordings. Main results and further investigations are finally discussed in the conclusion.

\section{MATHEMATICAL SKETCH}

Linear instantaneous BSS model assumes the existence of $n$ source signals $\left\{s_{i},(t), 1 \leq i \leq n\right\}$, statistically independent and zeromean and the observation of as many mixtures $\left\{x_{i}(t), 1 \leq i \leq\right.$ $n\}$, formed by linear combinations of the unknown sources, i.e. $x_{i}(t)=\sum_{j=1}^{n} a_{i j} s_{j}(t)$, for each $i=1, \ldots, n$. This is compactly represented by the mixing equation

$$
\boldsymbol{x}(t)=A s(t),
$$

where the matrix $A_{(n \times n)}$ collects the mixing coeficients, $s(t)=$ $\left(s_{1}(t), \ldots, s_{n}(t)\right)^{T}$ is a column vector collecting the source signals, $\boldsymbol{x}(t)$ collects the $n$ observed signals. ${ }^{T}$ denotes the transpose operator. The aim of BSS consists in retrieving the source vector $s(t)$ using only the observed data with the sole assumption of independence of the source signals. It can be reformulated as the computation of a $n \times n$ separating matrix $B$ whose output $\boldsymbol{y}(t)=B \boldsymbol{x}(t)$ is an estimate of the source signals $\boldsymbol{s}(t)$. Independence is much more than decorrelation. Performing a principal component analysis (PCA), i.e. decorrelating the outputs, is not sufficient for separating the sources (see [5]). In this paper, two BSS methods and wavelet denoising are briefly expounded and used on real ECG data and their performances are evaluated in terms of visual inspection.

\subsection{Stationary signal assumption}

For practical reasons, BSS algorithm are often separated in 2 parts [5]. One consists in sphering or whitening the data such that they become uncorrelated. The remaining part is then the estimation of a rotation and many algorithms have already been proposed for this purpose. One approach for estimating ICA consists in using higher-order cumulant tensors which are generalizations of the covariance matrix. We can use fourth-order cumulant tensors to cancel the fourth-order cumulants. Joint approximative diagonalization of eigenmatrices (JADE) refers to one principle of solving this kind of approximative higher-order decorrelation [4]. The eigenvectors give more or less directly the mixing matrix for whitened data. JADE code can be downloaded on Cardoso web page (http: //enst.fr/ cardoso).

\subsection{Nonstationary (NS) signal assumption}

Most of the approaches to BSS are implicitely based on a model where the sequence $s_{i}(t)$ is i.i.d., i.e. nothing is assumed concerning temporal evolution. We here examine the separation of fECG signals in the nonstationary case, i.e. falling the second ' $i$ ' in i.i.d. The idea is the following: a single covariance matrix does not give enough constraints to determine $A$, but a collection of several covariances matrix estimated over different time periods does determine $A$, provided the source distributions have changed enough over the whole observation period. The only assumption is that $s_{i}(t)$ and $s_{i}\left(t^{\prime}\right), t \neq t^{\prime}$, have different distributions. Details on the theoretical fundations can be found in $[13,15]$

In this approach, the objective function is derived by minimizing of the mutual information [15] between the vectors $\left[(B \boldsymbol{x})_{i}(1)\right.$, $\left.\ldots,(B \boldsymbol{x})_{i}(N)\right]^{T}, 1 \leq i \leq n$. Rather trying to estimate the actual mutual information, we shall consider the Gaussian mutual information defined as the ordinary mutual information with respect to some Gaussian random vectors with the same covariances structure as the random vector of interest. Denoting by $C(t)$ the covariance matrix of $\boldsymbol{x}(t)$, the normalized Gaussian mutual information equals $\frac{1}{T} \sum_{t=1}^{N}$ off $\left[B C(t) B^{T}\right]$. The quantity off $(A)$ of any positive matrix $A$ is a measure of deviation from diagonality $D(A)=\{A \mid \operatorname{diag}(A)\}$. The separation algorithm consists in minimizing $\frac{1}{T} \sum_{t=1}^{N}$ off $\left[B C(t) B^{T}\right]$ with respect to $B$. Since $C(t)$ varies slowly with $t$, one approximates the above criterion by $\frac{1}{L} \sum_{\ell=1}^{L}$ off $\left[B C\left(\frac{\ell N}{L}\right) B^{T}\right]$, which computation cost is lower. In [15], $C(t)$ is unknown, but estimated by a nonparametric kernel estimator, i.e.

$$
\hat{C}(t)=\frac{\sum_{\tau=1}^{N} \phi\left(\frac{t-\tau}{M}\right) \boldsymbol{x}(\tau) \boldsymbol{x}^{T}(\tau)}{\sum_{\tau=1}^{N} \phi\left(\frac{t-\tau}{M}\right)} .
$$

where $M$ is a window width parameter and $\phi(\cdot)$ a positive kernel function [15]. In the following, the algorithm will be referred as NS-joint diagonalisation algorithm.

\section{Block-on-line Algorithm}

The Gaussian mutual information approach can be easily turned into a block-on-line algorithm. The matrices $\hat{C}(t)$ can now be easily evaluated at any time point as a local average. The data stream is subdivided into data blocks of length $T$, for which the sample covariance matrix $\hat{C}(t)$ is computed as in Eq. (2). The separating matrix $B(t)$ is performed by jointly approximately diagonalizing the matrices $\hat{C}(t), \hat{C}(t-m), \ldots, \hat{C}(t+m(1-L))$ [15].

\section{WAVELETS FOR FECG DENOISING}

\subsection{Wavelet transform}

The wavelet transform (WT) [6]is a time-scale representation technique, which describes a signal by using the correlation with translation and dilatation of a function called "mother wavelet". Wavelet shape can be selected to match the shapes of components embedded in the signal to be analyzed. Such wavelets are excellent templates to separate those components and events from the analyzed signal waveform. The discrete wavelet transform (DWT) is a batch method, which analyses a finite-length time-domain signal at different frequency bands with different resolutions by successive decomposition into coarse approximation and detail information. Approximations represent the slowly changing features of the signal and conversely details represent the rapidly changing features of the signal.

\subsection{Wavelet de-noising}

The wavelet de-noising approach is based on the assumption that random errors in a signal are present over all the coefficients while 
deterministic changes get captured in a small number of relatively large coefficients. As a result, a nonlinear thresholding (shrinking) function in the wavelet domain will tend to keep a few larger coefficients representing the underlying signal, while the noise coefficients will tend to reduce to zero. Practically, the wavelet denoising method consists in applying the discrete wavelet transform to the original noisy data, thresholding the detail coefficients, and then inverse transforming the thresholded coefficients to obtain the time-domain de-noised data [14]. It should be noted that the performance of the wavelet de-noising depends to the choice of the thresholding rule, the type of wavelet, the maximum depth of wavelet decoposition and the initial SNR.

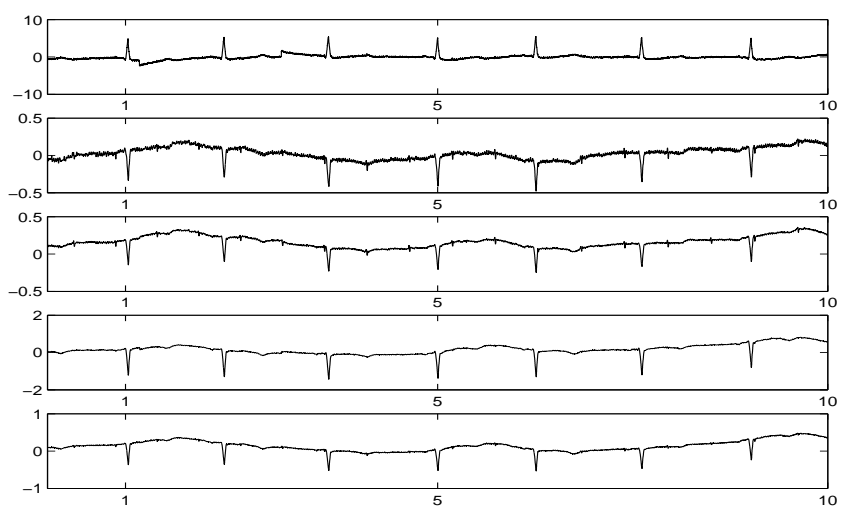

Figure 2: Five channels mother abdominal recording.

\section{APPLICATION TO REAL FECG DATA}

The fetal ECG (fECG) obtained from maternal abdomen normally has low amplitude and poor SNR, hence fetal heart rate can hardly be detected. The signal processing algorithms need to: $(i)$ remove the maternal ECG (mECG) complexes, $(i i)$ reduce the effect of motion artifact (mother breathing, uterine contraction, diaphragm, $50 \mathrm{~Hz}$ ) and (iii) enhance the fetal PQRST complexes.

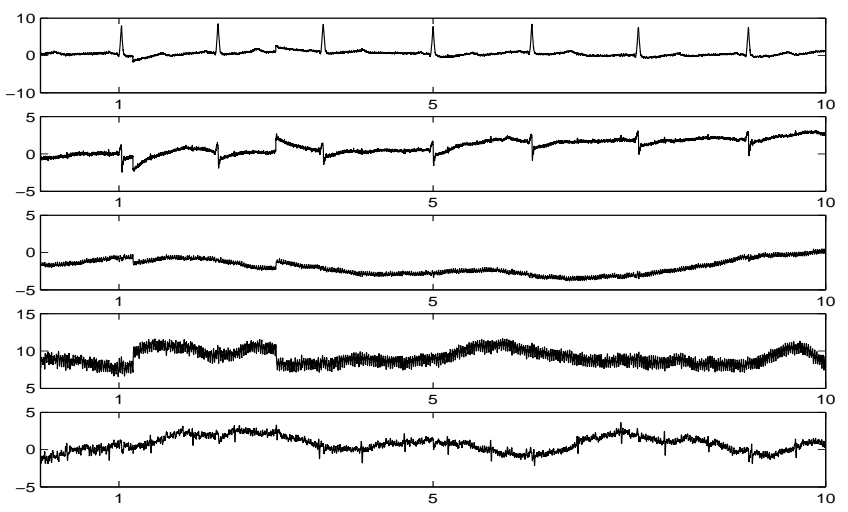

Figure 3: Estimated (separated) noisy source estimates with JADE algorithm.

The experimental data displayed Fig. 2 consist of a 5 channels experiment from a pregnant patient at the 28th week. Channels 1 to 5 show mother abdominal recorded potentials and contain weak fetal contributions. The horizontal axis displays the time in seconds. The vertical axis displays the amplitude values (in $m V$ ). The sampling frequency is $1 \mathrm{KHz}$, the number of samples $N=10000$, hence the duration of the signal is $10 \mathrm{~s}$. Even though the fetal ECG is much weaker than the maternal one, it is detectable. The source estimated with JADE algorithm are displayed in Fig. 3, and those estimated with the NS-joint diagonalization algorithm are displayed in Fig. 4. Channel 1 contains the noisy mECG signal while poor fECG is present in the signal on channel 5.

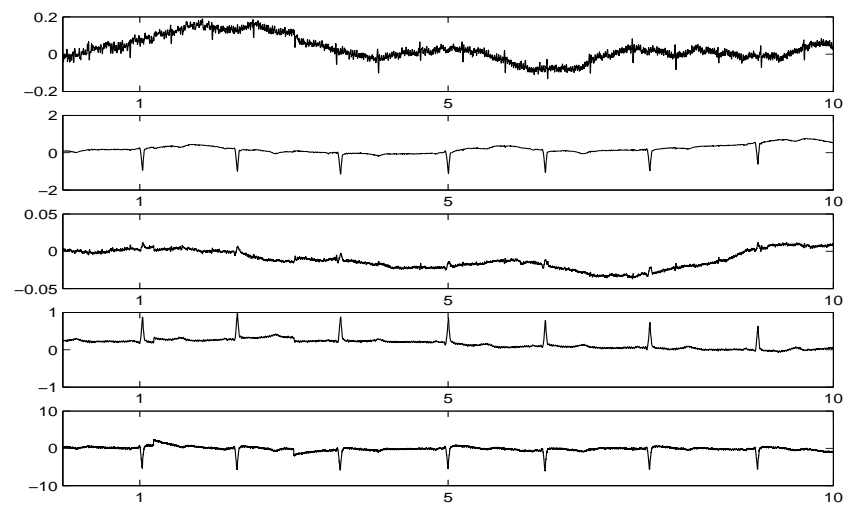

Figure 4: Estimated (separated) noisy source estimates with NSjoint diagonalization algorithm.

Baseline drift due to the mother breathing, electrode contacts or fetus movements make inspection of fECG difficult. The WT can be applied as a tool to obtain a good approximation of the fECG baseline (see also [19]). To this end, the wavelet decompositionreconstruction is performed with biorthogonal wavelets and only the approximation component between scales 1 and 7 (in the wavelet decomposition) is retained as signal carrying useful information (the 7-th scale component being the baseline estimation). Fig. 5 plots the fECG signal obtained after wavelet baseline removal stage. As the pattern of interest of fECG is the PQRST complex, removing the baseline provides a poor SNR and only wave R appears.

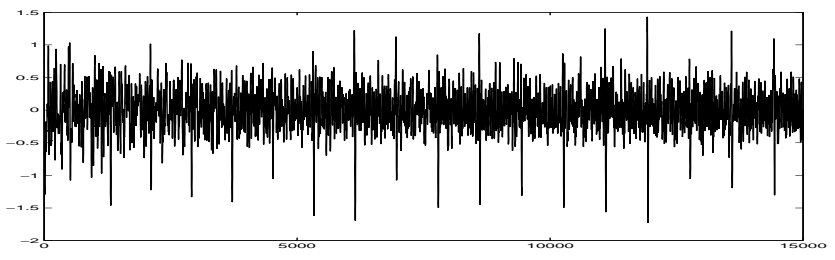

Figure 5: fECG obtained after wavelet baseline removal stage.

An improvement can be obtained by adding a processing step consisting in $(i) \mathrm{R}$-wave detection by determining the location of the relative maxima, (ii) PQRST amplification (duration of the PQRST interval was considered about 36,5 ms [7].

After PQRST amplification, a wavelet de-noising (for cancelling the noise) is applied to fECG signal with the following pa- 
rameters: biorthogonal wavelet type, decomposition level $\ell=6$, and the data-adaptive threshold selection rule SureShrink of MATLAB wavelet toolbox. Denoising is applied by blocs, each of 4096 samples. Fig. 6 plots the fECG signal obtained after wavelet baseline removal, PQRST amplification and wavelet de-noising stages. The set of processing is very efficient and the complete fetal PQRST complexes appear clearly (for visualisation reasons, only 3 PQRST complexes are shown).
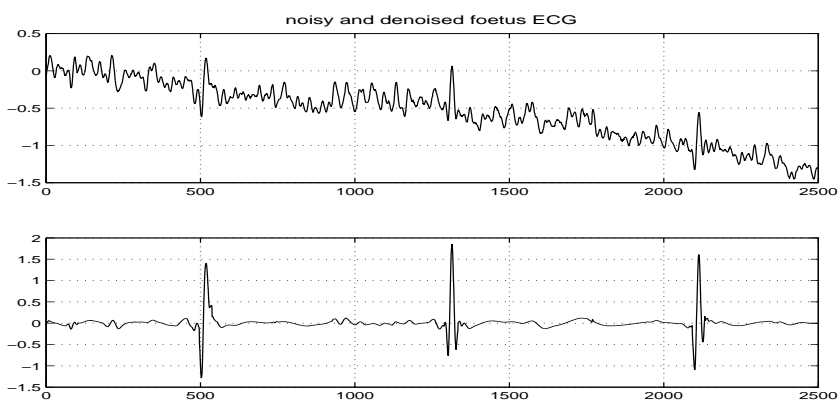

Figure 6: fECG obained after baseline removal, PQRST amplification and wavelet de-noising stages.

\section{CONCLUSIONS}

In this paper, we apply BSS to fECG extraction. We show that (i) exploiting the ECG non stationarity can improve source separation, (ii) wavelets seems a well-suited and promising method for extracting the fetal PQRST complexes. Further investigations will include (i) a quantitative comparison of performance of BSS algorithms and focus in particular on wavelet denoising for improving PQRST complex extraction, (ii) a qualitative evaluation (by physicians) of the fetal PQRST extracted by this method, especially in pathological cases.

\section{REFERENCES}

[1] S. Abboud, G. Barkai, S. Mashiach and D. Sadeh. Quantification of the fetal electrocardiogram using averaging technique, Comput. Biod. Med., 20, pp. 147-155, 1990.

[2] E. Bacharakis, A.K. Nandi and V. Zarzoso. Fetal ECG extraction using blind source separation methods.

[3] D. Callaerts. Signal separation based on singular value decomposition and their application to the real time extraction of the fetal electrocardiogram from cutaneous recordings. $\mathrm{Ph} . \mathrm{D}$. Thesis, E.E. Dept, KU Leuven, Decemder 1989.

[4] J.F. Cardoso. Multidimensional independent component analysis, Proc. ICASSP'98, 4, pp. 1941-19944, 1998.

[5] P. Comon. Independent Component Analysis, a new concept?. Signal Processing, Vol. 36, No 3, pp. 287-314, April 1994.

[6] I. Daubechies. Ten Lectures on Wavelets, Society for industrial and applied mathematics, 1992.

[7] R.S. Fowler and VC.D. Finlay. The electrocardiogram of the neonate. Bookchapter in The fetal circulation, pp. 72-80, 1978.
[8] C. Jutten, J. Herault, P. Comon. Blind separation of sources, Part I: An adaptive algorithm based on neuromimetic architecture. Part II / Problem statement, Signal Processing, 24, pp. 1-21, 1991

[9] A. Kam and A. Cohen. Maternal ECG elimination and fetal ECG detection algorithm ? Comparison of several algorithm, Proc. of the 20th Ann. Int. Conf. IEEE EMBS, Hong-Kong, 1998.

[10] L. de Lathauwer, B. de Moor and J. Vanderwalle. A singular value decomposition for higher-order tensors. Proc. ATHOS Workshop on System Identification and High-Order Statistics, Nice (France), pp. 20-21, 1983.

[11] H. Lilja, K. Larlsson, K. Lindecrantz, S.S. Ratnam, A.S. Thavarasah and K.G. Rosen, Microprocessor based waveform analysis of the fetal electocardiogram during labor, Int. J. Gynecol. Obstet., 30, pp. 109-116, 1989.

[12] C.J. Marvel, D.L. Kirk, H.M. Jenkins and E.M. Symonds. Normal condition of the fetal electrocardiogram during labour”, Br. J. Obstet. Gynaecol., 92, pp. 611-617, 1980.

[13] K. Matsuoka and M. Kawamoto. Blind signal separation based on Mutual information criterion, pp 95-91.

[14] A. Paraschiv-Ionescu, C. Jutten, K. Aminian, B. Najafi and $\mathrm{Ph}$. Robert. Source separation in strong noisy mixtures : a study of wavelet de-noising pre-processing, Proceed. of ICASSP'2002, Orlando (USA), May, 2002

[15] D.T. Pham and J.F. Cardoso. Blind Separation of Instantaneous Mixture of Nonstationary Sources, IEEE Trans. On SP, 49 (9), pp. 1837-1848, 2001.

[16] K.G. Rosen, R. Luzietti. Intrapartum fetal monitoring: basis and current developments, Prenat. Neonatal. Med., 9, pp. 114, 2001.

[17] O. Sibony, J.P. Fouillot, M. Bennaoudia, A. Benhala and P. Blot. Quantification of the fetal heart rate variability by spectral analysis of fetal well being and fetal distress, European Journal of Obstetrics and Gynaecology and Reproductive Biology, 54, pp. 103-108, 1994.

[18] C.N. Smyth, electrocardiography of the fetus, Lancet, 1124 1126, 1953.

[19] S. Thurner, M. C. Feuerstein and M. C. Teich. Multiresolution wavelet analysis of heartbeat intervals discriminates healthy patients from those with cardiac pathology, Phys. Rev. Lett., 80 (7), pp. 1544-1547, 1998. death, Cardiovasc. Res., 31, pp. 419-433, 1996.

[20] R. Vetter, J.M. Vesin and P. Celka. Observer of the Autonomic Cardiac Outflow in Humans using Non-causal Blind Source Separation, Proceedings of the First International Workshop on Blind source Separation and Independent Components Analysis (ICA'99), J.-F. Cardoso, C. Juten, Ph. Loubaton (Eds.) pp. 161-166, 1999.

[21] C. Widmark, T. Jansson, R. Lindecrantz and K.G. Rosen. ECG waveform short term heart rate variability and plasma cathecolamine concentrations in response to hypoxia in intra uterine growth retarded guinea pig fetuses, J. Develop. Physiol., 15, pp. 109-116, 1995.

[22] V. Zarzoso, A.K. Nandi and E. Bacharakis. Maternal and foetal ECG separation using blind source separation methods, MA Journal of Mathematics Applied in Medicine and Biology, 14(3), 1997. 